\title{
Neuropsychological alterations in borderline personality disorder: purpose of a case
}

\begin{abstract}
Different investigations reveal the neurological and neuropsychological differences among patients with BPD. The objective of this case study is to describe the neuropsychological (cognitive, executive), symptomatological and personality alterations of a patient with dual pathology and the implications that these alterations have in their daily lives. A battery of neuropsychological evaluation was applied to a patient undergoing treatment at the CEDRO Vigo drug dependence unit. The results reflect a neuropsychological performance indicative of a medium premorbid intelligence, a reduced speed of psychomotor and visuospatial processing, deficits in tasks of verbal memory, an executive functioning determined by alterations in the executive components of actualization, change and inhibition. As well as difficulties in social and work activity and a symptomatological profile characterized by obsessive-compulsive, anxious and psychotic clinical manifestations, an elevated motor and unplanned impulsivity, and a tendency to yield to positive affections and reduced perseverance. Neuropsychological exploration helps us to detect deficits in the cognitive / executive processes, generates awareness of the deficit and allows realizing a neuropsychological profile, which facilitates the design and elaboration of individualized treatment programs.
\end{abstract}

Keywords: borderline personality disorder, dual pathology, neuropsychological disorders, psychosocial functioning, neuropsychological exploration

\author{
Volume 9 Issue 6 - 2018
}

\author{
Torres-Rincón TM, , 1,2 Piñon-Blanco A, 1,3,4,5 \\ Vázquez-Justo $\mathrm{E}^{4,5}$ Otero-Lamas $\mathrm{F}^{1,3}$ \\ 'Assistance Unit Drug Addiction of the Concello deVigo \\ "CEDRO”, Spain \\ "University Hospital Complex of Vigo, Spain \\ ${ }^{3}$ Research Group on Psychiatric Diseases (IISGS), Research \\ Institute Sanitary Galicia South (IIS Galicia Sur), Spain \\ ${ }^{4}$ Portucalense Institute of Neuropsychology and Cognitive and \\ Behavioral Neurosciences (INPP), Portucalense University of \\ Porto, Portugal \\ ${ }^{5}$ Clinics ebam, Pontevedra, Spain
}

Correspondence: Adolfo Piñón Blanco, Assistance Unit for Drug Addiction of the Concello de Vigo CEDRO, Calle Pintor Colmeiro $N^{\circ} 9$, Post code: 3621 I Vigo-Pontevedra, Spain, Email Adalfo.pb@icloud.com

Received: August 24, 2017 | Published: November 28, 2018

\section{Introduction}

Borderline personality disorder (BPD) is characterized by the presence of affective instability, impulsivity, self-harm and severe dysfunction of interpersonal relationships. ${ }^{1}$ These psychopathological characteristics have centered the study of this disorder, being much less known and studied the neuropsychological deficits of these patients, whose study would allow us a better knowledge of this pathology, that includes new strategies in the planning of the psychotherapeutic and psychopharmacological treatment, as well as To orient ourselves on the patient's individual prognosis., ${ }^{2,3}$ Neuropsychological alterations in patients with psychiatric pathology have been studied, among others, in schizophrenia, ${ }^{4-8}$ obsessive-compulsive disorder, ${ }^{9,10}$ personality disorder, ${ }^{11,12}$ Personality disorder ${ }^{2,3}$ in substance-related disorders, ${ }^{13-18}$ autism, ${ }^{19}$ or attention deficit disorder. ${ }^{20,21}$ There is an important consensus in the scientific literature regarding the close relationship between neurocognition and psychosocial functioning in severe mental disorders, with negative interference from the former to the latter. $^{22,23}$

\section{Goals}

To describe the neuropsychological (cognitive, executive), symptomatological and personality alterations of a patient with dual pathologyandtheimplications thatthesealterationshaveintheirdailylife. To evaluate the presence of cognitive impairment through the exploration of basic cognitive processes (attention, memory and perception), executive functions (actualization, inhibition, cognitive flexibility and decision making), different components of impulsivity and symptomatology in the Time of evaluation.

\section{Presentation of the case}

$\mathrm{He}$ is a 41-year-old male, schooling until completing primary studies. Early onset of toxic use (at age 16) is reported, with high intravenous and inhaled cocaine use. It also presents antecedents of multiple serious autolytic attempts, as well as previous treatments in different devices of the area of drug addiction and in different modalities, from ambulatory to residential. At the time of the evaluation, it is included in a treatment program for psychosocial dependents (PTDP) in semi-voluntary mode (Day Unit), with diagnoses of Borderline Personality Disorder (F60.31 [301.83]) and Disorder (F14 [304.20]) according to the criteria of the fifth edition of the Diagnostic and Statistical Manual of Mental Disorders (DSM-V). ${ }^{24}$

\section{Material and method}

\section{Instruments}

An adaptation of the neuropsychological evaluation battery ${ }^{25}$ was applied and the patient evaluation protocol was administered in 2 sessions of 60 minutes duration.

\section{Instruments}

The evaluation of neuropsychology focused on assessing a possible cognitive impairment, with the assessment of basic cognitive processes (attention, memory and perception), executive functions (updating, inhibition, cognitive flexibility and decision making), different components of Impulsivity and symptomatology presented at the time of the evaluation. The tests used and the cognitive / executive domains evaluated (Table 1). 
Table I Tests used and cognitive domains/business

\begin{tabular}{|c|c|}
\hline Test & Cognitive domains / executives \\
\hline \multicolumn{2}{|l|}{ WAIS-III } \\
\hline Key Numbers & Psychomotor processing speed and hand-eye coordination \\
\hline Arithmetic & mental calculation and working memory \\
\hline vocabulary & As estimation of premorbid intelligence. Semantic memory \\
\hline digits & Focused and sustained attention \\
\hline Understanding & verbal understanding and social cognition \\
\hline search Symbols & visual perception, psychomotor speed processing \\
\hline \multicolumn{2}{|l|}{ Trail Making Test (TMT) } \\
\hline Part A & motor and visuo-spatial skills visual search \\
\hline \multicolumn{2}{|l|}{ test D2 } \\
\hline TR (processed Elements) & Psychomotor speed processing \\
\hline omissions & attentional control \\
\hline commissions & Inhibitory control \\
\hline CON (concentration) & Processing efficiency \\
\hline \multicolumn{2}{|l|}{ Stroop test } \\
\hline Words & Processing speed reader \\
\hline Colour & selective attention \\
\hline Word-Color & cognitive inhibition \\
\hline Verbal Learning Test (TAVEC) & verbal memory \\
\hline SCL-90 & symptomatic perception \\
\hline Barrat scale (BIS-I I) & Impulsiveness \\
\hline UPPS-P & Dimensions of impulsivity \\
\hline
\end{tabular}

\section{Results}

The results reflect a neuropsychological performance indicative of an average premorbid intelligence (WAIS-III Vocabulary, Centil=50), a reduced psychomotor processing speed (IVP WAIS-III, CI=81), and visuospatial processing (TMT-A $\mathrm{Pc}=25$ (TAVEC Recognition, $\mathrm{Sd}=-3$ ), an executive functioning determined by alterations in the executive components of updating (WAIT-III Arithmetic, Centil=16), change (STROOP Interference, $\mathrm{T}=36$ ) (SCL-90-R, Pc.95), anxious (SCL-90-R, Pc.95), and an inhibitory effect (SCO-90-R, Pc.95), a clinical profile characterized by obsessive- R, Pc.90) and psychoticism (SCL-90-R, Pc.85), a high in motor impulsivity (BIS-11/Pd=35) and unplanned impulsivity (BIS-11/Pd=26) Yielding to positive affects (UPPS-P, $\mathrm{Sd}=+1.76$ ) and reduced perseverance (UPPS-P, $\mathrm{Sd}=+1.42$ ); Alterations that make manifest in the day to day, in difficulties of voluntary control of the attention, reduced resistance to the interference, little tolerance to the cognitive effort, persevering behaviors, apathy and a reduced self-regulation of its behavior. On the UPPS scale, there is a tendency to give in to strong impulses when accompanied by positive emotions, a reduced capacity for perseverance and a high total impulsivity (Table 2). In Barrat's impulsivity scale, he scores high on motor and unplanned impulsivity (planning and organization of future actions) (Table 3). In the questionnaire assessing perceived subjective malaise (SCL-90-R), it presents a symptomatological profile characterized by clinical manifestations of obsessive-compulsive, anxious and psychotic (Table 4). In the specific processing speed tests, the average value of the Strooop ( $T=46)$, mid-low in the WAIS
III $(\mathrm{CI}=81)$ and below average In part A of the TMT (Pc.25) and the task of reaction time of D2 (Pc.10). Indicative of a reduced speed of psychomotor and visuospatial processing. With respect to attentional sub processes, it presents a reduced sustained and selective attention of internal character in tasks related to the adequate selection of which information is necessary to attend and which to ignore (StroopColores, T=32/D2 Omissions Pc.2) (Figure 1), so it can be said that it presents deficits in the sub processes of sustained, selective and alternating attention (cognitive flexibility). In particular, the deficits he presents are reflected in the difficulties he has in maintaining his attention when the required task is boring or monotonous, when he has to select the relevant information that must be addressed and the irrelevant to ignore, or When it must be cognitively flexible and must adapt its behavior to a novel or changing situation (Table 5).

Table 2 UPPS (impulsivity)

\begin{tabular}{lll}
\hline Negative urgency & & $\mathrm{Sd}=+0.9 \mathrm{I}$ \\
Lack of premeditation & $\mathrm{PD}=27$ & $\mathrm{Sd}=+0.80$ \\
Lack of Perseverance & $\mathrm{PD}=27$ & $\mathrm{Sd}=+1.42$ \\
Sensation Seeking & $\mathrm{PD}=33$ & $\mathrm{Sd}=+0.50$ \\
Positive urgency & $\mathrm{PD}=39$ & $\mathrm{Sd}=+1.76$ \\
Total & $\mathrm{PD}=159$ & $\mathrm{Sd}=+1.46$ \\
\hline
\end{tabular}


In the Verbal Memory Test (TAVEC), it achieves a medium-low performance in the processes of coding, storing, retrieving and evoking verbal information. This is reflected in a low-average performance in most of the test indices (free recall, learning curve, discrimination, recognition) and that is manifested in a reduced active recovery of information through the use of learning strategies. On the other hand, in the recognition test it obtains a medium-low score (-3sd), indicative of difficulties of access to information through associative recovery processes (keys) (Table 5).

Table 3 Barrat scale (BIS-II)

\begin{tabular}{ll}
\hline cognitive impulsivity & $\mathrm{PD}=23$ \\
motor impulsivity & $\mathrm{PD}=35$ \\
Unplanned impulsivity & $\mathrm{PD}=26$ \\
Total score & $\mathrm{PD}=84$ \\
\hline
\end{tabular}

Table 4 SCL-90

\begin{tabular}{lll}
\hline somatization & 1.42 & Pc.60 \\
Obsession / compulsion & 3.3 & Pc.95 \\
interpersonal sensitivity & 1,56 & Pc.65 \\
Depression & 2,38 & Pc.75 \\
Anxiety & 3 & Pc.90 \\
Hostility & 1.33 & Pc.65 \\
phobic anxiety & 1.71 & Pc.80 \\
paranoid ideation & $\mathrm{I}, 83$ & Pc.70 \\
psychoticism & 2.1 & Pc.85 \\
Symptomatic Board index & 2,08 & Pc.80 \\
Total positive symptoms & $7 \mathrm{I}$ & Pc.80 \\
Discomfort index & 2,63 & Pc.75
\end{tabular}

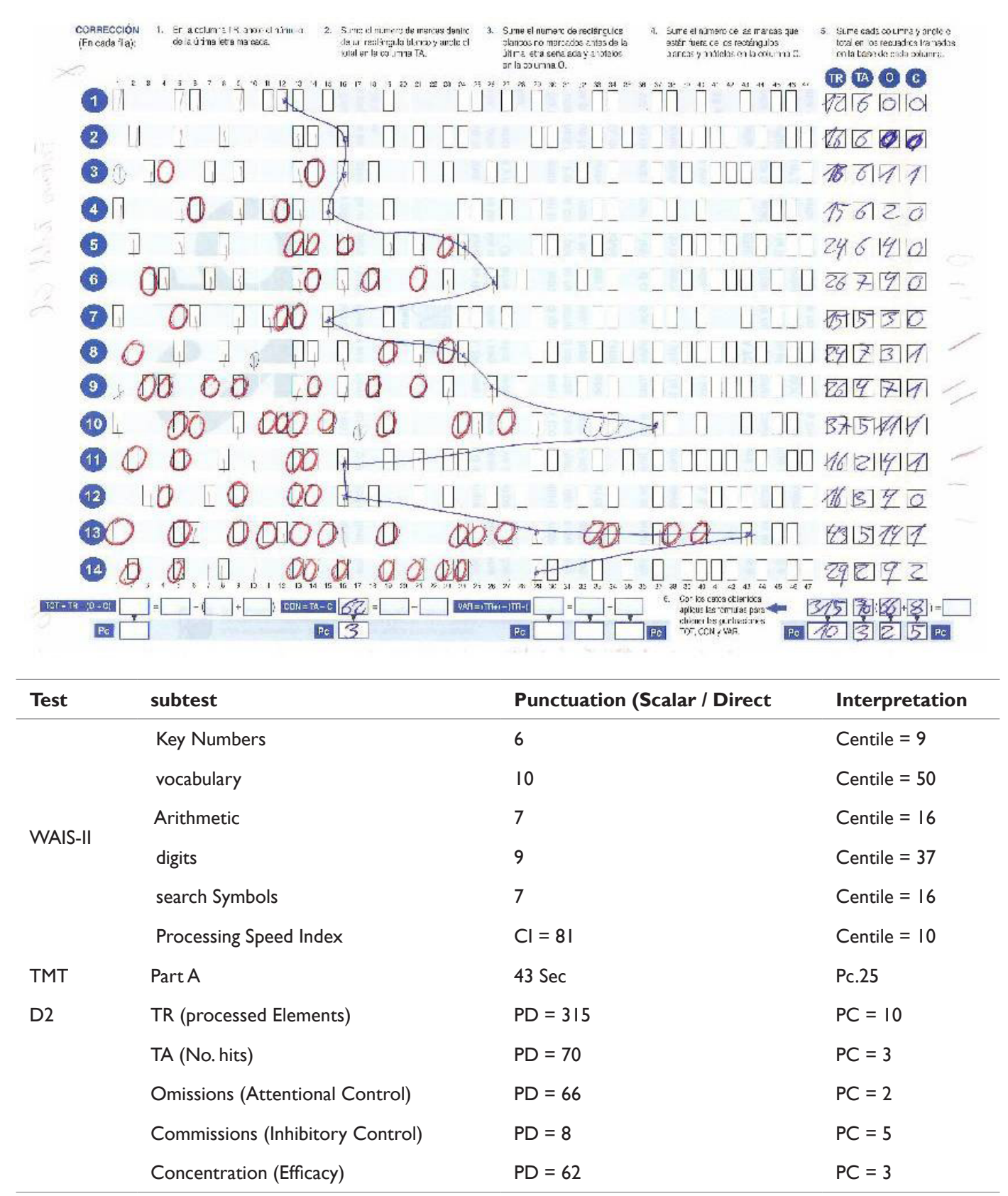




\begin{tabular}{llll}
\multicolumn{2}{l}{ Table Continued.... } & Punctuation (Scalar / Direct & Interpretation \\
\hline Test & subtest & $\mathrm{PD}=94$ & $\mathrm{~T}=46$ \\
\hline STROOP & Words & $\mathrm{PD}=53$ & $\mathrm{~T}=32$ \\
& Colors & $\mathrm{PD}=20$ & $\mathrm{~T}=20$ \\
& Word - Color & $\mathrm{PD}=-13$ & $\mathrm{~T}=36$ \\
& Interference & $4-6-7-8-7$ & $\mathrm{Sd}=-2$ \\
TAVEC & Learning (RLI) & $44 \%$ & $\mathrm{Sd}=+2$ \\
& Primacy & $9.5 \% / 9.5 \%$ & $\mathrm{Sd}=-4$ \\
& Half & $38 \%$ & $\mathrm{Sd}=+2$ \\
& recency & 12 & $\mathrm{Sd}=-3$
\end{tabular}

\section{Proposed treatment}

In order to improve the patient's adaptation to his / her environment, a cognitive rehabilitation program focused on techniques of restitution and compensation of attentional sub-processes and memory, as well as specific work on self-regulation of behavior (management of Emotions).

\section{User}

There are numerous publications linking prolonged use of illegal drugs with possible cognitive impairment. ${ }^{13-18}$ The results obtained in the exploration carried out are consistent with previous investigations that have shown a significant influence of the prolonged use of drugs on the functioning of different components of the executive function, ${ }^{18}$ as well as, with different investigations that show the Neurological and neuropsychological differences between patients with BPD and other psychiatric conditions. ${ }^{2,3}$ Although these results are consistent with previous studies, they should be interpreted with caution, since it is a dual population in which it is difficult to attribute the causal direction between neuropsychological alterations and psychopathology. However, the presence of these alterations supports the need to carry out neuropsychological rehabilitation programs in patients with BPD, especially those with comorbid substance abuse. The findings of this single case study should be interpreted with caution, taking into account the limitations of the studies in drug-dependent population, such as heterogeneous samples (polyconsumers, pattern of consumption), low level of cognitive reserve $^{17}$ And the difficulty in attributing the causal direction between neuropsychological alterations and prolonged substance use. Neuropsychological assessment has been shown to be a useful tool to help better define the needs for therapeutic intervention and to orient in a more efficient way the objectives of neurocognitive rehabilitation in the rehabilitative treatment of the drug-dependent population). ${ }^{26-29}$ Future prospective studies and large samples are needed to determine the possible effects of drug use on different components of executive functioning and their potential impact on the daily functioning of drug-dependent patients.

\section{Acknowledgments}

None.

\section{Conflicts of interest}

The author declares there is no conflicts of interest.

\section{References}

1. American Psychiatric Association. Diagnostic and statistical manual of mental disorders. $4^{\text {th }}$ ed. Washington, DC: American Psychiatric Association; 2000.

2. Arza R, Díaz-Marsá M, López-Micó L, et al. Neuropsychological alterations in borderline personality disorder: detection strategies. Actas Esp Psychiatry. 2009;37(4):185-190.

3. Arza R, Díaz-Marsá M, López-Micó L et al. Neuropsychological rehabilitation in patients with borderline personality disorder: a series of cases. Actas Esp Psychiatry. 2009;37(4):236-239.

4. Burton CZ, Harvey PD, Patterson TL, et al. Neurocognitive insight and objective cognitive functioning in schizophrenia. Schizophr Res. 2016;171(13):131-136.

5. Cuesta M, Peralta V, Zarzuela A. Cognitive alterations: a reality in schizophrenic disorder. Annals of Psychiatry. 1998;14:11-25.

6. Mother M, Canales-Rodríguez EJ, Ortiz-Gil J, et al. Neuropsychological and neuroimaging underpinnings of schizoaffective disorder: a systematic review. Acta Psychiatr Scand. 2016;134(1):16-30.

7. Heinrichs RW, Zakzanis KK. Neurocognitive deficit in schizophrenia: a quantitative review of the evidence. Neuropsychology. 1998;12(3):426-445.

8. Stip E. Cognition, schizophrenia and the effects of antipsychotics. Encéphale. 2006;31(3 Pt 1):341-350.

9. Torres A, Méndez L, Olivares JM, et al. Cognitive deficit and schizophrenia. Spanish Proceedings of Psychiatry. 2001;29(1):1-9.

10. Insel TR, Donnelly EF, Lalakea ML. Neurological and neuropsychological studies of patients with OCD. Biol Psychiatry. 1983;18(7):741-750.

11. Head D, Bolton D, Hymas N. Deficits in cognitive shifting ability in patients with OCD. Biological Psychiatry. 1989;25(7):929-937.

12. Deckel AW, Hesselbrock V, Basuer L. Antisocial personality disorder, childhood delinquency and frontal brain functioning: EEG and neuropsychological findins. Journal of Clinical Psychology. 1996;52(6):639-650.

13. Raine A, Buchsbaum M, La Casse L. Brain abnormalities in murderers indicated by PET. Biological Psychiatry. 1997;42(6):495-508.

14. Garavan H,Stout JC. Neurocognitive insights into substance abuse. Trends in Cognitive Sciences. 2005;9:195-201.

15. Fernández-Serrano MJ, Pérez-García M, Verdejo-García A, et al. What are the specific vs. Generalized effects of drugs on neuropsychological performance? Neurosci Biobehav Rev. 2011;35(3):377-406. 
16. Vázquez-Justo E, Vergara-Moragues E, Piñón-Blanco A, et al. Neuropsychological functioning in methadone maintenance patients with HIV. rev.latinoam.psicol. 2016;48(3).

17. Vázquez-Justo E, Piñón-Blanco A, Vergara-Moragues E, et al. Cognitive reserve during neuropsychological performance in HIV intravenous drug users. Applied Neuropsychology Adult. 2014;21(4):288-296.

18. Greenjo-Garcia A, Orozco Giménez C, Meersmans Sánchez-Jofré M, et al. Impact of the severity of drug use on different components of the executive function. Journal of Neurology. 2004;38:1109-1116.

19. Bennetto L. Intact and impaired memory functions in autism. Child Dev.1996;67(4):1816-1835.

20. Barkley RA. Behavioral inhibition, sustained attention and executive functions: constructing a unifying theory of ADHD. Psychol Bull. 1997;121(1):65-94

21. Dery M, Toupin J, Pauze R, et al. Neuropsychological characteristics of adolescents with conduct disorder: association with attention deficit hyperactivity and aggression. Journal of Abnormal Child Psychololy. 1999;27(3):225-236.

22. Green MF. What are the functional consequences of neurocognitive deficits in schizophrenia? Am J Psychiatry. 1996;153(3):321-330.

23. Penadés R, Catalán R, Puig O, et al. Executive function needs to be targeted to improve social functioning with Cognitive Remediation Therapy (CRT) in schizophrenia. Psychiatry Research. 2010;177(1-2):41-45.
24. American Psychiatric Association. Diagnostic and statistical manual of mental disorders. $5^{\text {th }}$ ed. Arlington, VA: American Psychiatric Publishing. 2013.

25. Sieira-Valiño J, Iglesias-Fungueiriño $M$, Sánchez Pérez $M$, et al. Neuropsychological battery for cognitive rehabilitation in drug dependence. Cadiz: Interdisciplinary Training Institute, University of Cadiz; 2011.

26. Schrimsher GW, Parker JD. Changes in cognitive function during substance use disorder treatment. Journal of Psychopathological and Behavioral Assessment. 2008;30:146-153.

27. Verdejo-García A, Bechara A. Neuropsychology and drug dependence: evaluation, clinical impact and applications for rehabilitation. In: Pérez García, editor. Manual of clinical neuropsychology. Madrid: Pyramid; 2009:179-208.

28. Aharonovich E, Hasin DS, Brooks AC, et al. Cognitive deficits predict low treatment retention in cocaine dependent patients. Drug Alcohol Depend. 2006;81(3):313-322.

29. Pace-Schott EF, Morgan PT, Malison RT, et al. Cocaine users differ from normals on cognitive tasks which show poor performance during drug abstinence. Am J Drug Alcohol Abuse. 2008;34(1):109-121. 